\title{
Explicit versus Implicit Processing of Representational Space in Neglect: Dissociations in Accessing the Mental Number Line
}

\author{
Konstantinos Priftis ${ }^{1,2}$, Marco Zorzi ${ }^{1}$, Francesca Meneghello ${ }^{2}$, \\ Roberto Marenzi ${ }^{3}$, and Carlo Umiltà ${ }^{1}$
}

\begin{abstract}
The present study investigated the effects of left hemispatial neglect on two tasks activating the mental number line (MNL). Six patients with left neglect performed a mental number bisection task and a modified version of the Spatial Numerical Association of Response Codes (SNARC) task. Effects of left neglect were observed in the number bisection
\end{abstract}

task, but not in the SNARC task. We argue that the dissociation between number bisection and SNARC resembles, in the representational space of the MNL, previously reported dissociations on neglect between explicit knowledge (assessed by direct tasks) and implicit knowledge (assessed by indirect tasks).

\section{INTRODUCTION}

Different studies reporting introspective descriptions (e.g., Seron, Pesenti, Noël, Deloche, \& Cornet, 1992; Galton, 1880), behavioral data, and neuroimaging data (for reviews, see Dehaene, Molko, Cohen, \& Wilson, 2004; Dehaene, Dehaene-Lambertz, \& Cohen, 1998) support the assumption of a continuous, analogical, and left-to-right-oriented mental number line (MNL) representing numbers, which is localized bilaterally in the intraparietal sulcus. The finding that the MNL obeys psychophysical laws governing sensory modalities cannot be explained by assuming a digital representation for basic number meaning. Participants' performance in number comparison is better when the distance between to-be-compared numbers increases (i.e., the numerical distance effect, Moyer \& Landauer, 1967) and, for equal numerical distance, performance worsens with increasing number magnitude (i.e., the number size effect, Moyer \& Landauer, 1967). Participants are faster at judging the parity of large numbers (e.g., 9) when responses are executed in the right hemispace, whereas they are faster at judging the parity of smaller numbers (e.g., 1) when responses are executed in the left hemispace (i.e., Spatial Numerical Association of Response Codes [SNARC], Dehaene, Bossini, \& Giraux, 1993). The SNARC effect suggests that relatively small number representations are spatially compatible with the left hemispace and that relatively large number representa-

${ }^{1}$ University of Padova, ${ }^{2}$ IRCCS San Camillo, Venezia-Lido, ${ }^{3}$ Centro di Riabilitazione di Conselve, Azienda Ospedaliera di Padova

tions are spatially compatible with the right hemispace (i.e., left-to-right-oriented MNL). Considered together, the numerical distance effect, the number size effect, and the SNARC effect suggest the existence of a continuous, analogical, and left-to-right-oriented MNL coding number magnitude and numerosity of perceived objects.

Zorzi, Priftis, and Umiltà (2002) tackled the issue of the spatial orientation of the MNL from a new perspective by exploring the representation of numbers in patients with hemispatial neglect. Patients with left neglect, following a right hemisphere lesion, fail to report, orient to, or verbally describe stimuli in the contralesional left hemispace (for reviews, see Halligan, Fink, Marshall, \& Vallar, 2003; Heilman, Watson, \& Valenstein, 1979). For instance, when they have to mark the midpoint of a linear segment positioned in front of them, they systematically displace the midpoint to a subjective position on the right of the true midpoint, as if they ignored the leftmost part of the presented linear segment. Furthermore, Halligan and Marshall (1988) reported that the rightward displacement is directly proportional to the length of the linear segment: The longer is the linear segment, the greater is the rightward displacement. However, a paradoxical phenomenon is observed for short linear segments, consisting in a leftward displacement of the midpoint (i.e., the crossover effect). In addition, similar effects of left neglect on bisection have been reported in studies involving the bisection of imaged linear segments (Chokron, Bernard, \& Michel, 1997; Bisiach, Rusconi, Peretti, \& Vallar, 1994; Ishiai et al., 1994). 
Zorzi et al. (2002) reasoned that, if the MNL were more than a metaphorical concept, neglect patients would have shown the same form of distortion in bisecting the MNL as they would show in bisecting perceived or imaged lines. To test this hypothesis, leftneglect patients were asked to perform a number bisection task that has been previously used to assess basic numerical skills in acalculic patients (Dehaene \& Cohen, 1997). Left-neglect patients were asked to indicate the midpoint of an orally presented number interval without performing mental calculations. Zorzi et al. reported a significant displacement of the midpoint of the number interval (e.g., Experimenter: "What number lies halfway between 1 and 9?" Patient: "7."). In addition, midpoint displacement was affected by the length of the number interval. That is, there was a progressive rightward displacement of the midpoint with increasing number intervals, except for the shortest intervals in which the crossover effect was reported. In contrast, the performance of right-brain-damaged patients without neglect and healthy participants was intact. It is important to emphasize that neglect patients had intact numerical and arithmetical skills. This is consistent with the observation that number processing deficits (i.e., acalculia) typically originate from lesions of the inferior parietal region of the language-dominant hemisphere (for a review, see Dehaene, Piazza, Pinel, \& Cohen, 2003).

This new form of representational neglect was discussed by Zorzi et al. (2002) in terms of a functional isomorphism between MNL and visual lines. These findings were replicated by Rossetti et al. (2004), who, in addition, showed that the disrupted performance of neglect patients in mental number interval bisection was ameliorated by a short adaptation to rightwarddeviating prisms. In the same vein, Vuilleumier, Ortigue, and Brugger (2004) showed that neglect patients were slower in processing the "leftmost" number in a number comparison task.

A way to further explore the phenomenon described by Zorzi et al. (2002) is to make recourse to the distinction between implicit knowledge (assessed by direct tasks) and explicit knowledge (assessed by indirect tasks) of contralesional stimuli, which is one of the most intriguing phenomena in the literature concerning neglect (for a review, see Berti, 2002). Left-neglect patients demonstrate spared unconscious (implicit) processing of information that is ignored at the conscious (explicit) level (Làdavas, Palladini, \& Cubelli, 1993; Berti \& Rizzolatti, 1992; Marshall \& Halligan, 1988).

The number bisection task can be considered as a direct task that requires voluntary access of the spatial frame of the MNL, presumably through the orientation of spatial attention. This is confirmed by the fact that neglect patients reported performing the task by forming a left-to-right-oriented visuospatial representation of the numbers composing the interval and by subsequently attempting to locate the midpoint. The task, however, does not allow one to establish whether neglect disrupts the MNL or rather produces a bias related to the access and exploration of an intact MNL.

The aim of the present study was to compare the effects of left neglect in a number interval bisection task and in a modified version of the SNARC task (Fias, Brysbaert, Geypens, \& d'Ydewalle, 1996; Dehaene et al., 1993). In the SNARC task, number magnitude is task-irrelevant because participants respond to the parity of centrally presented Arabic numerals. However, the SNARC effect demonstrates that the MNL's spatial frame is activated indirectly and automatically (Fias et al., 1996; Dehaene et al., 1993). Note that the number interval bisection task and the SNARC task tap into the same number representation (i.e., a spatially oriented MNL). This notion is shared by most, if not all, researchers of numerical cognition (for recent reviews, see Fias \& Fischer, 2005; Hubbard, Piazza, Pinel, \& Dehaene, 2005). Therefore, if the dissociation between explicit and implicit processing holds in the case of the representational space of the MNL, left neglect should affect number interval bisection (i.e., the task requiring direct activation of the left-to-right-oriented spatial frame of the MNL), but not the SNARC effect (i.e., the byproduct of the indirect activation of the MNL). A dissociation between the bisection task and the SNARC task would therefore allow us to discriminate between a deficit in accessing an intact MNL or a deficit in the representation of the MNL itself. A further aim of the present study was to test the reliability of the results of the Zorzi et al. (2002) study in a new, unselected sample of neglect patients.

\section{EXPERIMENT 1: NUMBER INTERVAL BISECTION IN LEFT-NEGLECT PATIENTS}

\section{Methods}

Participants

Six patients with left neglect following right hemisphere stroke participated in the study, after giving their informed consent according to the Declaration of Helsinki (mean age, 66 years; mean education, 9 years). Peripersonal neglect (i.e., neglect within reaching space) was assessed through a standardized battery (Behavioral Inattention Test; Wilson, Cockburn, \& Halligan, 1987). Extrapersonal neglect (i.e., neglect beyond reaching space) was assessed by verbal description and by pointing toward objects in the neuropsychological assessment room. Selective omissions of objects in the left extrapersonal space were considered as a sign of extrapersonal neglect. Demographic, clinical, and psychometric data of left-neglect patients are reported in Table 1. Participants had virtually intact cognitive functions, such as overall cognitive status, short-term auditory memory (i.e., digit span), immediate and delayed long-term verbal learning, semantic verbal fluency, verbal reasoning, and nonverbal 
Table 1. Demographic, Clinical, and Psychometric Data of Patients with Left Neglect

\begin{tabular}{|c|c|c|c|c|c|c|c|c|c|c|c|c|}
\hline & \multicolumn{6}{|c|}{ Neglect } & \multicolumn{6}{|c|}{ Controls without Neglect } \\
\hline & F.B. & P.D.P. & P.L.P & $R . R$ & A.T. & B.C. & $O . M$. & E.B. & A.I. & P.M. & $L . Z$. & M.P. \\
\hline Sex & $\mathrm{F}$ & $\mathrm{F}$ & $\mathrm{F}$ & M & $\mathrm{F}$ & M & $\mathrm{F}$ & M & M & M & M & M \\
\hline Age (years) & 70 & 70 & 67 & 67 & 63 & 60 & 70 & 72 & 66 & 61 & 60 & 65 \\
\hline Education (years) & 5 & 13 & 8 & 8 & 5 & 13 & 5 & 8 & 5 & 13 & 5 & 8 \\
\hline Handedness & $\mathrm{R}$ & $\mathrm{R}$ & $\mathrm{R}$ & $\mathrm{R}$ & $\mathrm{R}$ & $\mathrm{R}$ & $\mathrm{R}$ & $\mathrm{R}$ & $\mathrm{R}$ & $\mathrm{R}$ & $\mathrm{R}$ & $\mathrm{R}$ \\
\hline Lesion site & FTP & $\mathrm{BN}$ & $\mathrm{FP}$ & $\mathrm{BN}$ & $\mathrm{C}$ & $\mathrm{TP}$ & $\mathrm{BN}$ & $\mathrm{C}$ & FTP & FTP & $\mathrm{BN}$ & $\mathrm{FTt}$ \\
\hline Lesion etiology & IS & IS & IS & HS & IS & IS & IS & HS & IS & IS & HS & HS \\
\hline BIT $^{\mathrm{a}}$ (cutoff: $129 / 146$ ) & 139 & 116 & 58 & 121 & 119 & 136 & 140 & 136 & 140 & 146 & 138 & 145 \\
\hline Extrapersonal neglect ${ }^{\mathrm{b}}$ & + & + & + & + & + & + & - & - & - & - & - & - \\
\hline $\mathrm{MMSE}^{\mathrm{C}}$ & $30 / 30$ & $26 / 30$ & $26 / 30$ & $28 / 30$ & $29 / 30$ & $30 / 30$ & $23 / 30$ & $28 / 30$ & $28 / 30$ & $30 / 30$ & $28 / 30$ & $26 / 30$ \\
\hline \multicolumn{13}{|l|}{ Digit $\operatorname{span}^{\mathrm{d}}$} \\
\hline Forward & 5 & 7 & 6 & 8 & 6 & 6 & 4 & 6 & 5 & 6 & 5 & 6 \\
\hline Backward & 5 & 4 & 4 & 5 & 4 & 3 & 2 & 4 & 2 & 5 & 3 & 4 \\
\hline Semantic verbal fluency ${ }^{\mathrm{e}}$ (cutoff: $0 / 4$ ) & $2 / 4$ & $4 / 4$ & $3 / 4$ & $2 / 4$ & $4 / 4$ & $3 / 4$ & $2 / 4$ & $4 / 4$ & $2 / 4$ & $4 / 4$ & $2 / 4$ & $2 / 4$ \\
\hline Verbal reasoning ${ }^{\mathrm{f}}$ (cutoff: $0 / 4$ ) & $1 / 4$ & $2 / 4$ & $1 / 4$ & $3 / 4$ & $3 / 4$ & $2 / 4$ & $1 / 4$ & $4 / 4$ & $2 / 4$ & $4 / 4$ & $2 / 4$ & $2 / 4$ \\
\hline \multicolumn{13}{|l|}{ Verbal memory (Rey 15 Words) ${ }^{g}$} \\
\hline Immediate recall (cutoff: 28.53) & 43 & 52.1 & 44 & 30 & 30.3 & 43.4 & 45.2 & 34.9 & 32.1 & 41.6 & 37.4 & 46.9 \\
\hline Delayed recall (cutoff: 4.69) & 9.4 & 13.2 & 9.3 & 5.3 & 6.7 & 8.2 & 9.8 & 7.9 & 7.8 & 11 & 8.2 & 8.7 \\
\hline $\begin{array}{l}\text { Raven Progressive Matrices ' } 47 \mathrm{~g} \\
\quad(\text { cutoff: } 18.96)^{\mathrm{h}}\end{array}$ & 29.5 & 15.1 & 21 & 24.6 & 22.7 & 23.4 & 24 & 36.6 & 17.9 & 32.8 & NA & NA \\
\hline \multicolumn{13}{|l|}{ Mathematical Screening } \\
\hline Oral counting & $4 / 4$ & $4 / 4$ & $4 / 4$ & $4 / 4$ & $4 / 4$ & $4 / 4$ & $4 / 4$ & $4 / 4$ & $4 / 4$ & $4 / 4$ & $4 / 4$ & $4 / 4$ \\
\hline Parity judgement & $22 / 22$ & $22 / 22$ & $22 / 22$ & $20 / 22$ & $22 / 22$ & $22 / 22$ & $21 / 22$ & $22 / 22$ & $22 / 22$ & $22 / 22$ & $22 / 22$ & $22 / 22$ \\
\hline Number comparison & $14 / 14$ & $14 / 14$ & $14 / 14$ & $14 / 14$ & $14 / 14$ & $14 / 14$ & $13 / 14$ & $14 / 14$ & $14 / 14$ & $14 / 14$ & $14 / 14$ & $14 / 14$ \\
\hline Number words writing & $25 / 25$ & $25 / 25$ & $25 / 25$ & $25 / 25$ & $25 / 25$ & $25 / 25$ & $22 / 25$ & $25 / 25$ & $25 / 25$ & $25 / 25$ & $25 / 25$ & $25 / 25$ \\
\hline Arabic digit writing & $23 / 25$ & $25 / 25$ & $25 / 25$ & $25 / 25$ & $25 / 25$ & $25 / 25$ & $24 / 25$ & $25 / 25$ & $25 / 25$ & $25 / 25$ & $25 / 25$ & $25 / 25$ \\
\hline Arabic digit reading & $17 / 22$ & $21 / 22$ & $21 / 22$ & $22 / 22$ & $22 / 22$ & $22 / 22$ & $22 / 22$ & $22 / 22$ & $22 / 22$ & $22 / 22$ & $22 / 22$ & $22 / 22$ \\
\hline Operation signs & $4 / 4$ & $4 / 4$ & $4 / 4$ & $4 / 4$ & $4 / 4$ & $4 / 4$ & $4 / 4$ & $4 / 4$ & $4 / 4$ & $4 / 4$ & $4 / 4$ & $4 / 4$ \\
\hline Oral multiplications & $7 / 10$ & $8 / 10$ & $9 / 10$ & $9 / 10$ & $8 / 10$ & $10 / 10$ & $5 / 10$ & $9 / 10$ & $9 / 10$ & $10 / 10$ & $10 / 10$ & $10 / 10$ \\
\hline Oral additions & $8 / 10$ & $10 / 10$ & $10 / 10$ & $10 / 10$ & $9 / 10$ & $10 / 10$ & $10 / 10$ & $10 / 10$ & $10 / 10$ & $10 / 10$ & $10 / 10$ & $10 / 10$ \\
\hline Oral subtractions & $10 / 10$ & $10 / 10$ & $10 / 10$ & $10 / 10$ & $10 / 10$ & $10 / 10$ & $9 / 10$ & $10 / 10$ & $10 / 10$ & $10 / 10$ & $10 / 10$ & $10 / 10$ \\
\hline Oral repetitions & $16 / 16$ & $16 / 16$ & $16 / 16$ & $16 / 16$ & $16 / 16$ & $16 / 16$ & $16 / 16$ & $16 / 16$ & $16 / 16$ & $16 / 16$ & $16 / 16$ & $16 / 16$ \\
\hline \multirow{2}{*}{\multicolumn{13}{|c|}{$\begin{array}{l}\mathrm{M}=\text { male; } \mathrm{F}=\text { female; } \mathrm{BN}=\text { basal nuclei; } \mathrm{F}=\text { frontal; } \mathrm{T}=\text { temporal; } \mathrm{P}=\text { parietal; } \mathrm{C}=\text { capsular; } \mathrm{t}=\text { thalamus; } \mathrm{R}=\text { right; } \mathrm{IS}=\mathrm{ischemic} \text { stroke; } \\
\mathrm{HS}=\text { hemorrhagic stroke; }(+)=\text { deficit present; }(-)=\text { deficit absent; } \mathrm{BIT}=\text { Behavioral Inattention Test; } \mathrm{NA}=\text { not available. } \\
\text { a Wilson et al. (1987). }\end{array}$}} \\
\hline & & & & & & & & & & & & \\
\hline \multicolumn{13}{|c|}{ berbal description and pointing to objects located in the neuropsychological evaluation room. } \\
\hline \multicolumn{13}{|l|}{${ }^{\mathrm{c}}$ Folstein, Folstein, and Mc Hugh (1975). } \\
\hline \multicolumn{13}{|l|}{ dOrsini and Laicardi (1997). } \\
\hline \multicolumn{13}{|l|}{${ }^{\mathrm{e}}$ Novelli et al. (1986). } \\
\hline \multicolumn{13}{|l|}{ fSpinnler and Tognoni (1987). } \\
\hline${ }^{\mathrm{g}}$ Carlesimo, Caltagirone, Gainotti, and $\mathrm{Nc}$ & entini $(1$ & 1995). & & & & & & & & & & \\
\hline ertical version in order to av & & & & & & & & & & & & \\
\hline
\end{tabular}


reasoning (except for patient P.D.P.). Patients' numerical and mathematical abilities were perfect or near-perfect, as shown in Table 1.

The performance of neglect patients was compared with that of two control groups. The first group comprised six healthy participants with no history of neurological and psychiatric disorders (mean age, 67 years; mean education, 12 years). The second group was composed of six right hemisphere patients without neglect (mean age, 66 years; mean education, 7 years; see Table 1).

\section{Design}

A repeated measures design was used. The predictor variable was the number interval length (four levels: 3, 5, 7 , and 9). The criterion variable was the mean arithmetic difference between reported and correct responses (reported - correct).

\section{Stimuli}

Stimuli consisted of pairs of spoken number words (e.g., "one-three," "one-five," "one-seven," etc.). Each pair defined a specific number interval. All possible number intervals, having an integer as midpoint, were selected (e.g., 1-3, midpoint $=2$ ), whereas number intervals having a numerical fraction as midpoint were excluded (e.g., 1-2, midpoint $=1.5$ ). Each number pair included one of four possible number intervals with a length of three (e.g., 1-3), five (e.g., 1-5), seven (e.g., 1-7), or nine (e.g., 1-9). The same number intervals were repeated within the units (i.e., single digits from 1 to 9; e.g., 1-7), the teens (i.e., numbers from 11 to 19; e.g., 11-17), and the twenties (i.e., numbers from 21 to 29 ; e.g., 21-27). The final set of stimuli comprised 48 number pairs subdivided into 16 pairs within the units, 16 pairs within the teens, and 16 pairs within the twenties.

\section{Procedure}

Forty-eight spoken number pairs were presented randomly to the participants. Following oral presentation of each number interval, participants were asked to orally report the number lying halfway between the first number and the second number in the presented pair (e.g., Experimenter: "Which number is halfway between 1 and 9?'). There was no time limit for responding, and number pairs were repeated following participants' request. The whole task was administered for a second time using a backward presentation of the 48 forward trials, so as to counterbalance order effects (e.g., Experimenter: "Which number is halfway between 9 and 1?"). Given that the correct response could be obtained by computing, for example, the arithmetic mean of the numbers composing the number pair, participants were explicitly asked to avoid arithmetic calculations. Otherwise, putative MNL processing distortions due to left neglect could be bypassed by an alternative arithmetic procedure (e.g., calculation of the mean) and, consequently, such distortions would not be observed during the number interval bisection task. Note that left-neglect patients were not explicitly asked to use visuospatial imagery in order to solve the number interval bisection task.

\section{Results}

For each patient, the mean difference between observed $(O)$ and correct $(C)$ responses $(\mathrm{d} O-C)$ was computed for every number interval length. A preliminary linear regression analysis revealed a positive relation between number interval length and mean deviation from true midpoints $(\mathrm{d} O-C)$. For number interval lengths of 5,7 , and 9, the deviation to the right of the midpoint increased as a function of number interval length (see Figure 1). In contrast, there was a leftward deviation for the smallest interval (3) (i.e., the crossover effect; e.g., Experimenter: "What number is halfway between 6 and 8?" Patient: "6.").

Individual data were analyzed through a regression procedure for repeated measures designs (Method 3; Lorch \& Myers, 1990). For each participant, we performed a regression analysis, with length of the interval as the predictor variable, to calculate individual regression slopes. Then, one-sample $t$ tests were performed to test whether regression slopes ( $\beta$ weights) of

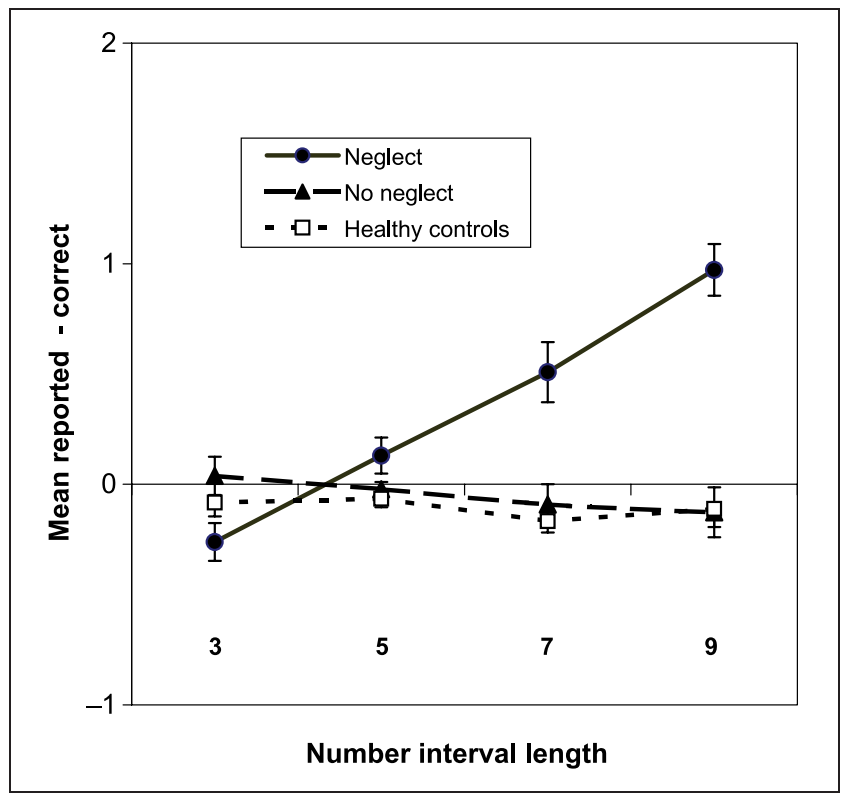

Figure 1. MNL bisection in neglect patients and in controls. The graph shows the difference between a reported and a correct midpoint as a function of interval length. Zero indicates the correct response (midpoint), positive values indicate shifts to the right, and negative values indicate shifts to the left. 
the group (neglect patients or controls) deviated significantly from zero. Finally, the slopes of neglect patients and controls were compared with a two-sample $t$ test. Note that the modulating effect of length would be revealed by a positive slope, indicating that misplacement increases with increasing lengths (as in Zorzi et al., 2002).

Regression slopes of neglect patients, mean $\beta=.20$, $S E=0.030$, were significantly different from zero, $t(5)=$ $6.18, p<.001$, one-tailed (see Figure 1). In contrast, the performance of controls was not affected by number interval length, healthy controls: mean $\beta=-.01$, $S E=0.009, t(5)=-1.332, n s$; control patients without neglect: mean $\beta=-.04, S E=0.054, t(5)=0.458$, $n s$. The direct comparison of participants' slopes was highly significant, $F(2,15)=13.33, p<.001$, two-tailed. Post hoc comparisons (with Bonferroni corrections) showed that the slopes of neglect patients were significantly different from those of healthy controls $(p<$ $.01)$ and control patients without neglect $(p<.001)$. In contrast, the slopes of healthy controls and control patients without neglect were not significantly different.

To further analyze the crossover effect shown by neglect patients, the mean $\mathrm{d} O-C$ for the shortest number interval length (i.e., 3) was calculated for each patient. Subsequently, the mean deviation was tested against zero with a one-sample $t$ test. The results showed that the leftward deviation for the shortest interval was significant, $t(5)=3.04, p<.05$, one-tailed.

\section{Discussion}

The present group study replicated the findings of Zorzi et al.'s (2002) multiple single-case study. Neglect patients showed a highly consistent error pattern in bisecting the MNL. For the larger number intervals (i.e., 5, 7, and 9), there was a progressive shift to the right of the midpoint with increasing interval length. In contrast, the MNL's midpoint was significantly shifted to the left for the shortest interval (i.e., 3).

During follow-up, neglect patients systematically reported that they performed the bisection task by visualizing a left-to-right-oriented representation of the numbers within the interval and by subsequently attempting to establish the midpoint. Counting numbers before and after the subjective midpoint was frequently reported, but only as a control procedure. Thus, response bias likely arises during visualization of the MLN because all neglect participants had excellent counting abilities. Note that, in the instructions, there was no mention of the possibility of forming visual images of the numbers. Participants also reported that they complied with the instructions of avoiding any calculations (e.g., taking the mean of the two given numbers).

The observed effect of number interval length on an MNL bisection resembles the well-documented perform- ance of left-neglect patients in bisecting perceived lines of increasing physical length (Halligan \& Marshall, 1988), in bisecting imaged lines (Chokron et al., 1997; Bisiach et al., 1994; Ishiai et al., 1994), or in mental scanning of familiar places (e.g., landmarks on the left or on the right of Piazza Duomo in Milan; Bisiach \& Luzzatti, 1978). In conclusion, the results of Experiment 1 are in accordance with the claim of Zorzi et al. (2002) that the MNL has visuospatial properties similar to those of perceived lines.

However, as already pointed out in the Introduction, number bisection is a direct task that requires explicit access and manipulation of the MNL. It is conceivable that the left sector of the number interval to be mentally bisected is omitted or compressed: For instance, "7" should appear as a plausible midpoint instead of 5 for the interval " $1-9$ " if the space between 1 and 5 is compressed, or if some of the numbers are missing. Alternatively, attention might be captured by numbers on the right sector of the mental number interval (i.e., 6-9), leading to a rightward response bias. It would seem interesting to ask whether the same effects could be observed in an indirect task, such as the SNARC task, which does not require explicit access and manipulation of the MNL.

\section{EXPERIMENT 2: SNARC IN HEALTHY PARTICIPANTS}

\section{Introduction}

In the standard SNARC procedure, the participants' body midline is aligned with the midline of the computer screen, and stimuli are presented in the center of the display. This might be a problem with left-neglect patients, who are severely impaired in visuospatial processing and are likely to miss a number of stimuli even if centrally presented. Second, in the standard SNARC procedure, participants use both hands in order to execute keypress responses. However, the majority of left-neglect patients is affected by partial (i.e., hemiparesis) or complete (i.e., hemiplegia) motor disorders that make accurate control of their left hand virtually impossible. Thus, in left-neglect patients, responses can be executed only with an intact right hand. In brief, there are problems in the use of the standard SNARC paradigm with left-neglect patients, which concern both the input (i.e., limited visuospatial processing) and the output (i.e., inability to use the left hand).

Therefore, a new version of the SNARC task was conceived, wherein the computer screen was positioned entirely within the right intact hemispace and participants executed their responses only with their right hand, using the index finger and the middle finger. Given that both stimuli and responses take place in the right hemispace, the term Unilateral SNARC Paradigm (USP) was coined (see Figure 2). 


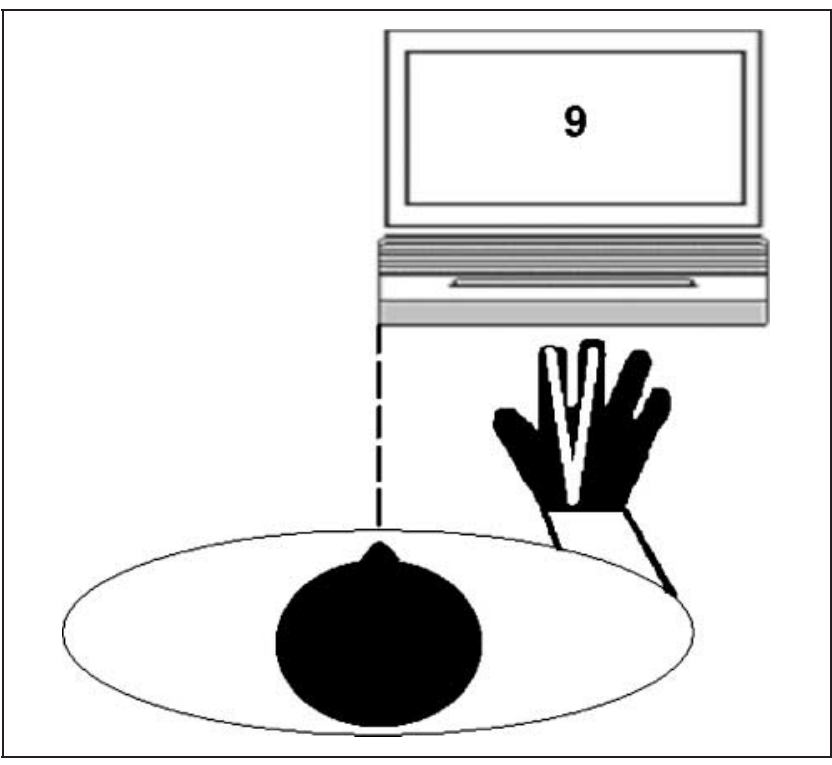

Figure 2. The USP. The computer screen was located in the right hemispace of the participant. Responses were executed using the index and middle fingers of the right hand.

Note that the SNARC effect might depend on the coding of effectors in egocentric coordinates (i.e., with reference to the participant's body midline). If this were the case, this new version of the task would not produce an SNARC effect. In contrast, if the effectors were coded in relative terms (i.e., the index finger being on the relative left of the middle finger, even though both belong to the same response hand), we should observe the SNARC effect even with the USP. The importance of relative coding of the effectors is suggested by Experiment 6 of Dehaene et al. (1993), in which a regular SNARC effect was observed even if participants responded with their hands crossed.

\section{Methods}

\section{Participants}

Sixteen undergraduate students of the University of Padova ( 8 men and 8 women; mean age, 25.1 years; range, 21-35 years) participated in this experiment. Fourteen students were right-handed, whereas two were ambidextrous. They had normal or corrected-to-normal vision. All participants were volunteers and naïve about the purpose of the experiment.

\section{Design}

Experimental variables were manipulated within a repeated measures design (Fias et al., 1996). The predictor variable was the number magnitude, whereas the criterion variable was the difference between the reaction time (RT) of the index finger and the RT of the middle finger: $\mathrm{dRT}=\mathrm{RT}$ (middle finger) $-\mathrm{RT}$ (index finger) Positive dRTs indicate faster responses with the leftmost effector, whereas increasingly negative dRTs indicate faster responses with the right effector.

\section{Apparatus and Stimuli}

The experiment was created and controlled using PsychLab software, version 2.2.7 (Gum \& Bub, 1988). Experimental stimuli were displayed on an 11-in., 256-color screen of a Macintosh Powerbook computer (central processing unit clock, $1400 \mathrm{~Hz}$; random access memory, $93.4 \mathrm{Mb}$ ), supporting a screen resolution of $800 \times$ 640 pixels (screen refresh rate, $60 \mathrm{~Hz}$ ). Each trial comprised three stimuli. The first stimulus was a fixation cross measuring $1.6 \times 1.6 \mathrm{~cm}$. The second stimulus was an Arabic digit (range, 1-9; 5 was excluded) measuring $0.9 \mathrm{~cm}$ in height and $0.5 \mathrm{~cm}$ in width. Finally, the third stimulus was the word "ready," measuring $1.8 \mathrm{~cm}$ in height and $3.8 \mathrm{~cm}$ in width. All experimental stimuli were created using Macintosh Geneva black fonts (type size, 36 points), and they were presented in the center of the screen against a white background.

\section{Procedure}

The experiment took place in a quiet, dimly lit room without environmental distractions. The experimenter aligned the trunk midline of each participant with the left border of the computer. The viewing distance was about $50 \mathrm{~cm}$. Next to this, participants were instructed to place their right hand on the keyboard. More precisely, the index finger was placed on key $\mathrm{B}$ (on the left half of the keyboard), whereas the middle finger was placed on key N (on the right half of the keyboard; USP; see Figure 2).

First, the fixation cross was displayed for $400 \mathrm{msec}$, followed by an interstimulus interval of $500 \mathrm{msec}$. Second, an Arabic digit appeared at fixation for $600 \mathrm{msec}$, and participants were asked to judge its parity by pressing key $\mathrm{N}$ or key B. Acoustic feedback was provided following response execution. That was a high-frequency tone for correct responses or, alternatively, a low-frequency tone for incorrect responses. Finally, after a responsestimulus interval of 1000 msec, the word "ready" appeared and remained on until the participant was ready and the next trial had started. The intertrial interval had a duration of 1500 msec.

Participants were tested individually in a single session that comprised two experimental conditions: the "oddeven" condition and the "even-odd" condition. In the odd-even condition, participants were asked to respond to odd digits with their index finger and to respond to even digits with their middle finger. This assignment was reversed in the even-odd condition, where participants were asked to respond to even digits with their index finger and to respond odd digits with their middle 
finger. Half of the participants performed the odd-even condition first, followed by the even-odd condition, whereas the other half performed the opposite sequence. Each experimental condition comprised a training block of 24 trials followed by an experimental block of 104 trials.

\section{Results}

Errors and responses faster than $150 \mathrm{msec}$ or slower than 2500 msec were excluded from statistical analyses (4\% of the total). A preliminary regression analysis showed a negative relation between the predictor variable (number) and the criterion variable (dRT), revealing the presence of the SNARC effect (see Figure 3A).

Data were further analyzed with a regression procedure for repeated measures designs (Method 3; Lorch \& Myers, 1990). First, 16 individual regression analyses were performed, one for each participant. Then, obtained slopes (regression $\beta$ coefficients) were compared against zero with a one-sample $t$ test. The analysis revealed that the participants' regression slopes were

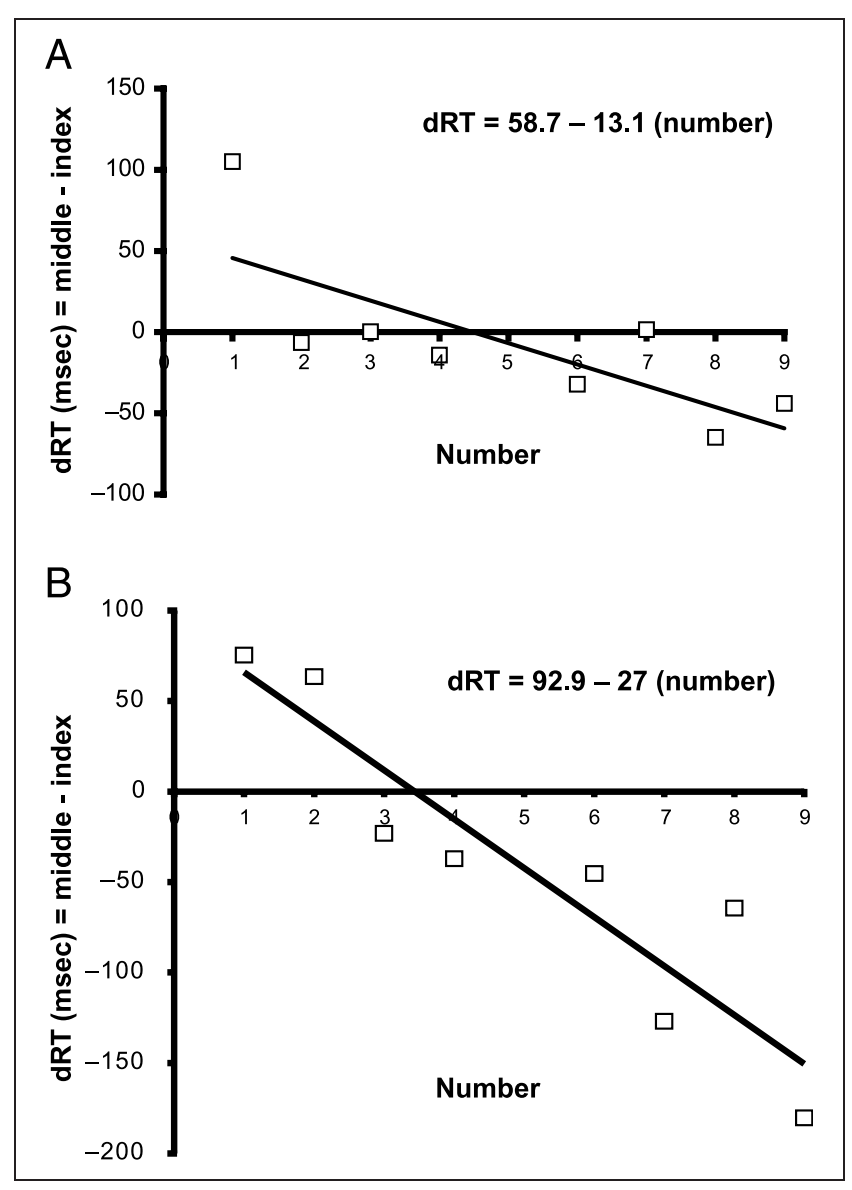

Figure 3. Regression equation and plot showing a negative correlation between number magnitude and the mean difference between the index and the middle finger $(\mathrm{dRT}[\mathrm{msec}]=\mathrm{RT}$ middle - RT index). (A) Healthy participants; (B) neglect patients. significantly different from zero, $t(15)=-6.22, p<.001$, one-tailed.

\section{Discussion}

The SNARC effect was observed in this preliminary study using the USP. There was a significant negative relation between number magnitude and processing speed. More precisely, there was a relative left effector advantage (index finger) in processing small numbers, which progressively shifted to a relative right effector advantage (middle finger) for larger numbers (see Figure 3A). Two conclusions can be derived from these results: First, the USP paradigm reveals the abstract and flexible nature of the MNL, suggesting that the SNARC is not dependent on egocentric coordinates and can be observed even when both stimuli and effectors are within one hemispace; second, the USP allows one to study the SNARC effect in left-neglect patients who might control efficiently only half of their peripersonal hemispace or who can use only their ipsilesional hand.

\section{EXPERIMENT 3: SNARC IN LEFT-NEGLECT PATIENTS}

\section{Methods}

\section{Participants}

The same left-neglect patients who had taken part in Experiment 1 participated in the present experiment.

\section{Design, Apparatus, Stimuli, and Procedure}

The same design, apparatus, stimuli, and procedure as in Experiment 2 were used. The only difference was that each session was performed in two consecutive days to avoid effects of fatigue and distractibility, which are common in left-neglect patients.

\section{Results}

The same data analyses were carried out as in Experiment 2. Errors and responses faster than $150 \mathrm{msec}$ or slower than 2500 msec were excluded from further analyses (11\% of total). Overall regression analysis showed a negative correlation between the predictor (number) and the criterion (dRT), similar to that shown by the healthy participants of Experiment 2 (see Figure 3B).

Individual regression slopes of neglect patients differed significantly from zero, $t(5)=3.7, p<.001$, onetailed, suggesting the presence of a regular SNARC effect. A direct comparison between healthy participants and neglect patients (Experiment 2 vs. Experiment 3) was performed by comparing the slopes and intercepts of both groups. Welch's $t$ tests were used in order to 
control variance dishomogeneity. Neither comparison was significant, slopes: $t(5.9)=1.9, n s$, two-tailed; intercepts: $t(5.5)=0.64, n s$, two-tailed.

\section{Discussion}

Left-neglect patients showed a regular SNARC effect. They were more efficient in responding to small numbers (range, 1-4) with the relative left effector (i.e., the index finger), whereas they were more efficient in responding to larger numbers (range, 6-9) with the relative right effector (i.e., the middle finger). If the relative left half of the MNL (i.e., 1-4) were impaired, no spatial-numerical compatibility should have been observed for these numbers (e.g., a flat regression line for the range 1-4). This pattern was not observed, suggesting that the spatial frame of the MNL was intact.

Furthermore, performance of left-neglect patients was not significantly different from that of the considerably younger health participants: Both groups showed a negative correlation between number magnitude and the space where the response was executed (i.e., the SNARC effect).

\section{GENERAL DISCUSSION}

In the Introduction, it was highlighted that left neglect selectively affects the explicit processing of contralesional information in direct tasks, leaving intact the implicit processing of the same information in indirect tasks (e.g., Làdavas et al., 1993; Berti \& Rizzolatti, 1992; Marshall \& Halligan, 1988). The aim of the present research was to investigate the role of different task demands upon the representational space of the MNL. To do so, we compared the performance of left-neglect patients in two numerical tasks that impose different processing demands: number interval bisection versus SNARC. Number interval bisection requires direct access and exploration of the MNL. In contrast, in the SNARC task, accessing the MNL is not an explicit task demand, and responses do not require any manipulation of number magnitude (i.e., of the MNL). Nonetheless, the MNL manifests itself because it is automatically activated during the parity judgement task. Indeed, there is evidence that encoding of spatial information can be strongly influenced by explicit task demands. For instance, Bachtold, Baumuller, and Brugger (1998) showed that the SNARC effect depended on the type of spatial frame imposed on numerical stimuli by experimental instructions. When the instructions were to imagine numbers along a ruler (i.e., a frame congruent with the MNL), there was a regular SNARC effect. In contrast, when the instructions were to imagine numbers on an analogical clock face, participants were faster in processing small numbers with their right hand (e.g., hours earlier than 6 o'clock) and were faster in processing large numbers with their left hand (e.g., hours later than 6 o'clock).

Performance of left-neglect patients in our study was impaired only in number interval bisection (direct task), whereas it was intact in the SNARC task (indirect task). It could be argued that the dissociation reflects the activation of different numerical representations in the two tasks, rather than a dissociation between implicit and explicit processing of the MNL. However, the consensus view in the numerical cognition literature is that the number bisection task and the SNARC task tap into the same number representation (i.e., a spatially oriented MNL; for recent reviews, see Fias \& Fischer, 2005; Hubbard et al., 2005).

Our findings, besides replicating and corroborating the results of Zorzi et al. (2002), show that neglect produces a deficit in directly accessing an intact MNL, rather than a deficit in the representation of the MNL itself. More generally, they demonstrate that the dissociation between explicit and implicit processing in neglect can be extended to a representational space-the MNL.

\section{Acknowledgments}

This study was supported by grants from the MIUR (PRIN 2003 to C. U. and PRIN 2004 to M. Z.) and the European Commission (Marie Curie Research Training Network's "Numeracy and Brain Development" to M. Z.). We thank all participants of the study and the personnel of IRCCS San Camillo, LidoVenice and Centro di Riabilitazione di Conselve, Azienda Ospedaliera di Padova; Valeria Colavito, Antonia Fumarola, and Anastassia Gavridou for their excellent assistance in data collection; and two anonymous reviewers for their helpful comments.

Reprint requests should be sent to Konstantinos Priftis, Dipartimento di Psicologia Generale, via Venezia 8, 35131 Padua, Italy, or via e-mail: konstantinos.priftis@unipd.it.

\section{REFERENCES}

Bachtold, D., Baumuller, M., \& Brugger, P. (1998). Stimulus-response compatibility in representational space. Neuropsychologia, 36, 731-735.

Berti, A. (2002). Unconscious processing in neglect. In H. O. Karnath, A. D. Milner, \& G. Vallar (Eds.), The cognitive and neural bases of spatial neglect (pp. 313-326). Oxford, UK: Oxford University Press.

Berti, A., \& Rizzolatti, G. (1992). Visual processing without awareness: Evidence from unilateral neglect. Journal of Cognitive Neuroscience, 4, 345-351.

Bisiach, E., \& Luzzatti, C. (1978). Unilateral neglect of representational space. Cortex, 14, 129-133.

Bisiach, E., Rusconi, M. L., Peretti, V. A., \& Vallar, G. (1994). Challenging current accounts of unilateral neglect. Neuropsychologia, 11, 1431-1434.

Carlesimo, G. A., Caltagirone, C., Gainotti, G., \& Nocentini, U. (1995). Batteria per la valutazione del deterioramento mentale: II. Standardizzazione e affidabilità diagnostica nell'identificazione di pazienti affetti da sindrome demenziale. Archivio di Psicologia, Neurologia e Psichiatria, 4, 471-488. 
Chokron, S., Bernard, J. M., \& Michel, I. (1997). Length representation in normal and neglect subjects with opposite reading habits studied through a line extension task. Cortex, 1, 47-64.

Dehaene, S., Bossini, S., \& Giraux, P. (1993). The mental representation of parity and number magnitude. Journal of Experimental Psychology: General, 122, 371-396.

Dehaene, S., \& Cohen, L. (1997). Cerebral pathways for calculation: Double dissociation between rote verbal and quantitative knowledge of arithmetic. Cortex, 33, 219-250.

Dehaene, S., Dehaene-Lambertz, G., \& Cohen, L. (1998). Abstract representations of numbers in the animal and human brain. Trends in Neurosciences, 21, 355-361.

Dehaene, S., Molko, N., Cohen, L., \& Wilson, A. J. (2004). Arithmetic and the brain. Current Opinion in Neurobiology, 14, 1-7.

Dehaene, S., Piazza, M., Pinel, P., \& Cohen, L. (2003). Three parietal circuits for number processing. Cognitive Neuropsychology, 3, 487-506.

Fias, W., Brysbaert, M., Geypens, F., \& d'Ydewalle, G. (1996). The importance of magnitude information in numerical processing: Evidence from the SNARC effect. Mathematical Cognition, 2, 95-110.

Fias, W., \& Fischer, M. H. (2005). Spatial representation of numbers. In J. I. D. Campbell (Ed.), Handbook of mathematical cognition (pp. 43-54). New York: Psychology Press.

Folstein, M. F., Folstein, S. E., \& Mc Hugh, P. R. (1975). Mini Mental State: A practical method for grading the cognitive state of patients for the clinician. Journal of Psychiatric Research, 3, 189-198.

Galton, F. (1880). Visualised numerals. Nature, 21, 252-256.

Gum, T., \& Bub, D. (1988). PsychLab. Montreal: Montreal Neurological Institute.

Halligan, P. W., Fink, G. R., Marshall, J. C., \& Vallar, G. (2003). Spatial cognition: Evidence from visual neglect. Trends in Cognitive Sciences, 3, 125-133.

Halligan, P. W., \& Marshall, J. C. (1988). How long is a piece of string? A study of line bisection in a case of visual neglect. Cortex, 2, 321-328.

Heilman, K. M., Watson, R. T., \& Valenstein, E. (1979). Neglect and related disorders. In K. M. Heilman \& E. Valenstein (Eds.), Clinical neuropsychology (pp. 268-307). New York: Oxford University Press.
Hubbard, E. M., Piazza, M., Pinel, P., \& Dehaene, S. (2005). Interactions between number and space in parietal cortex. Nature Reviews Neuroscience, 6, 435-448.

Ishiai, S., Sugishita, M., Watabiki, S., Nakayama, T., Kotera, M., \& Gono, S. (1994). Improvement of left unilateral spatial neglect in a line extension task. Neurology, 2, 294-298.

Làdavas, E., Paladini, R., \& Cubelli, R. (1993). Implicit associative priming in a patient with left visual neglect. Neuropsychologia, 12, 1307-1320.

Lorch, R. F., \& Myers, J. L. (1990). Regression analyses of repeated measures data in cognitive research. Journal of Experimental Psychology: Learning, Memory, and Cognition, 1, 149-157.

Marshall, J. C., \& Halligan, P. W. (1988). Blindsight and insight in visuo-spatial neglect. Nature, 336, 766-767.

Moyer, R. S., \& Landauer, T. K. (1967). Time required for judgments of numerical inequality. Nature, 215 , 1519-1520.

Novelli, G., Papagno, C., Capitani, E., Laiacona, M., Vallar, G., \& Cappa, S. F. (1986). Tre test clinici di ricerca e produzione lessicale: Taratura su soggetti normali. Archivio di Psicologia, Neurologia e Psichiatria, 4, 477-506.

Orsini, A., \& Laicardi, C. (1997). WAIS-R. Contributo alla Taratura Italiana. Firenze: Organizzazioni Speciali.

Rossetti, Y., Jacquin-Courtois, S., Rode, G., Ota, H., Michel, C., \& Boisson, D. (2004). Does action make the link between number and space representation? Visuo-manual adaptation improves number bisection in unilateral neglect. Psychological Science, 6, 426-430.

Seron, X., Pesenti, M., Noël, M. P., Deloche, G., \& Cornet, J. A. (1992). Images of numbers, or "When 98 is upper left and 6 sky blue". Cognition, 44, 159-196.

Spinnler, H., \& Tognoni, G. (1987). Standardizzazione e taratura italiana di test neuropsicologici. Italian Journal of Neurological Sciences, 8, 1-120.

Vuilleumier, P., Ortigue, S., \& Brugger, P. (2004). The number space and neglect. Cortex, 40, 399-410.

Wilson, B., Cockburn, J., \& Halligan, P. W. (1987). The Behavioural Inattention Test. Bury St. Edmunds, UK: Thames Valley Test Company.

Zorzi, M., Priftis, K., \& Umiltà, C. (2002). Brain damage: Neglect disrupts the mental number line. Nature, 417 , 138-139. 